\title{
The Link Between Periodontal Disease and Rheumatoid Arthritis: An Updated Review
}

\author{
Joanna Koziel • Piotr Mydel • Jan Potempa
}

Published online: 24 January 2014

(C) The Author(s) 2014. This article is published with open access at Springerlink.com

\begin{abstract}
Porphyromonas gingivalis is a leading pathogen in chronic periodontitis, a disease process involving progressive destruction of the tissues that support the teeth. Recently, the organism has been reported to produce a unique bacterial enzyme, $P$. gingivalis peptidyl-arginine deiminase (PPAD), which has the ability to convert arginine residues in proteins to citrulline. Protein citrullination alters protein structure and function; hence, PPAD may be involved in deregulation of the host's signalling network and immune evasion. Further, accumulating evidence suggests a role for autoimmunity against citrullinated proteins in the development of rheumatoid arthritis (RA). As inflammatory conditions in the lungs of cigarette smokers contribute to the breakdown of immune tolerance to citrullinated epitopes, chronic exposure to citrullinated proteins at periodontitis sites may also predispose susceptible individuals to the development of autoantibodies and the initiation of RA. In this review, we discuss evidence that PPAD may represent a mechanistic link between periodontitis and RA, diseases that are known to be significantly associated at the epidemiological level.
\end{abstract}

This article is part of the Topical Collection on Rheumatoid Arthritis

J. Koziel $(\bowtie) \cdot$ J. Potempa

Department of Microbiology, Faculty of Biochemistry, Biophysics, and Biotechnology, Jagiellonian University, ul. Gronostajowa 7, 30-387 Krakow, Poland

e-mail: joanna.koziel@uj.edu.pl

J. Potempa

e-mail: jan.potempa@louisville.edu

P. Mydel

Broegelmann Research Laboratory, The Gade Institute,

University of Bergen, Bergen, Norway

e-mail: Piotr.Mydel@k2.uib.no

J. Potempa

Oral Health and Systemic Diseases Research Group,

University of Louisville School of Dentistry, Louisville, KY, USA
Keywords Periodontal disease $\cdot$ Rheumatoid arthritis · Citrullination $\cdot$ PPAD $\cdot$ Periodontitis $\cdot$ P gingivalis peptidyl-arginine deiminase (PPAD)

\section{Introduction}

Rheumatoid arthritis (RA) and periodontal disease (PD) are two common chronic inflammatory diseases affecting humans worldwide. In 2003, the total cost attributable to arthritis and other rheumatic conditions in the United States was $\$ 128$ billion, equivalent to $1.2 \%$ of the 2003 U.S. gross domestic product (http://www.cdc.gov/arthritis/data_statistics). Furthermore, $23 \%$ of the US population age $65+$ has been reported to suffer from severe PD. Together, development of both diseases brings considerable consequences for public health and for the quality of life of affected individuals.

There may be a non-causal association between PD and RA due to shared genetic and environmental risk factors, such as expression of the MHC class II HLA-DRBI allele and smoking, respectively [1-5]. Despite differences in initiating etiological mechanisms, evidence emerging from numerous clinical and epidemiological studies suggests an association between RA and PD [6-9]. Compared to the general population, subjects with PD are at an increased risk of developing $\mathrm{RA}$, and vice versa; PD is at least 2-fold more prevalent in patients with RA. In addition, the clinical course of PD in RA patients is more severe and is independent of age, gender, ethnicity, or smoking history, as compared to non-RA individuals. Furthermore, RA and PD utilise similar effector destructive mechanisms, in that the inflammatory cells and proinflammatory cytokines that drive chronic bone erosion in RA and chronic gum destruction in PD are similar. Current novel and exciting findings strongly support the idea that PD could be a factor in the initiation and maintenance of the autoimmune inflammatory responses that occur in RA [10]. 


\section{Porphyromonas gingivalis is a Key Pathogen in Periodontitis}

Periodontitis, the chronic inflammation of the supporting tissues surrounding the teeth, is one of the most prevalent inflammatory diseases of mankind. Tooth loss is common in severe forms of the disease and has been reported to afflict more than $20 \%$ of the human population [11]. Further, a paradigm is emerging linking periodontitis with the development of atherosclerosis [12] and RA [7, 9, 13, 14].

It is now generally accepted that chronic periodontitis is initiated by the colonisation of dental plaque by a set of pathogenic bacteria, especially $P$. gingivalis, Tannerella forsythia, and Treponema denticola. These periodontal pathogens, referred to as the "red complex" due to their strong association with the disease [15], are well equipped with a broad array of virulence factors. $P$. gingivalis expresses lipopolysaccharide (LPS), fimbrae, and haemagglutins, which enable the bacterium to colonise and invade periodontal pockets, and is therefore known as the "master manipulator" of the host homeostatic system [16]. Its most potent weapons are, however, extracellular cysteine proteases, referred to as gingipains, which allow the bacterium to use the host innate immune response to its own benefit $[17,18]$. Because its gingipains render it resistant to complement, P.gingivalis directly benefits from activation of the complement pathway, which initiates and maintains the inflammatory reaction [19]. In addition, degradation of antimicrobial peptides by gingipains allows other pathogenic bacteria co-aggregating with $P$. gingivalis to persist in the gingiva. Moreover, gingipains affect proinflammatory signalling pathways by cleavage and activation of the proteinase-activated receptor-2 (PAR-2) on human neutrophils. Futile attempts by the host immune response to eliminate infection subsequently lead to connective tissue damage, including alveolar bone resorption $[20,21]$.

\section{Role of Citrullination in RA Development}

It is now clear that the majority of RA cases are triggered by an autoimmune response to citrullinated proteins. Such proteins are generated under physiological conditions, but the loss of tolerance in genetically susceptible individuals initiates the generation of autoantibodies against citrullinated proteins (ACPA) in the synovia and the subsequent development of RA [22-24].

Protein citrullination by enzymatic deimination of the guanidine group of an arginine side chain is the post-translational modification that converts positively charged peptidylarginine to neutral peptidyl citrulline. Protein citrullination is essential for many physiological processes, including terminal differentiation of the epidermis (pro-filaggrin and keratin), brain development [myelin basic protein (MBP)], and regulation of gene expression via chromatin remodeling $[25,26]$.
Apart from being involved in many physiological processes, citrullination also occurs under the pathological inflammatory conditions of excessive cellular apoptosis, necrosis, and netosis. In the latter process, hypercitrullination of histones is needed for formation of neutrophil extracellular traps, which are part of the innate immune system response to bacterial infection [26]. Thus, deimination has been linked to multiple sclerosis, psoriasis, Alzheimer's disease, primary open-angle glaucoma, obstructive nephropathy, and RA.

The citrullination process leads to alterations in intra- and inter-molecular interactions of protein targets possessing Arg residues essential for their structure. This may alter the threedimensional architecture of modified proteins and their solubility in water, and may lead to the generation of neo-epitopes, thus breaching immunological tolerance to citrullinated proteins. In susceptible individuals, the process may initiate a cascade of events leading to the induction of RA [22, 23, 27]. Antibodies to ACPA are known to be a sensitive and specific marker that can be detected years before the clinical onset of the disease. During the clinical course of RA, their presence and serum levels strongly correlate with disease severity. A recent report reveals elevated levels of ACPA in patients with aggressive periodontitis [28]. Furthermore, RA patients diagnosed as ACPA-positive were more likely to have moderate to severe periodontitis than ACPA-negative RA patients [29]. Because the ACPA response is peptidyl citrulline-specific, host enzymes which catalyse the protein modifications that lead to the generation of citrullinated epitopes are considered highly important targets for drug development.

\section{PPAD is a Unique Bacterial Enzyme among Peptidyl-arginine Deiminases}

Protein citrullination is carried out by peptidyl-arginine deiminases (PADs; enzyme commission number (EC) 3.5.3.15). The activity of mammalian PAD enzymes is dependent on high concentrations of calcium $\left(\mathrm{Ca}^{2+}\right)$; thus, citrullination is likely to occur in conditions that lead to the mobilisation of free intracellular calcium, such as chemokine receptor ligation, cell death, and differentiation. Five different PADs (PAD1, 2, 3, 4, and 6) identified in humans are encoded by five paralogous genes clustered on chromosome 1p35-36. PAD1 and PAD3 are mainly found in epidermis and hair follicles; PAD2 is expressed in a variety of tissues, including muscle, brain, and haematopoietic cells; and human PAD4 (formerly known as PAD5) is found primarily in haematopoietic cells. PAD homologues for some or all of these enzymes have also been found in other mammals, with similar genomic organisation across species [30].

$P$. gingivalis is unique among periodontal pathogens in its expression of peptidyl-arginine deiminase (PPAD). Some enzymatic properties of PPAD differ significantly from those of 
the human PADs. Specifically, PPAD is active at a higher $\mathrm{pH}$ and does not require calcium for activity. PPAD also citrullinates C-terminal arginine residues and deiminates free arginine, while mammalian PADs lack these activities [31]. The process of modification of C-terminal arginines is facilitated by colocalisation of PPAD in the outer membrane with arginine-specific gingipains (RGPs), cysteine proteases of P. gingivalis $[32 \bullet \cdot]$. Gingipains cleave protein chains, exposing C-terminal arginines that are rapidly citrullinated by PPAD, which generates polypeptides with citrullinated Arg located at the C-terminus. Arg-gingipain-null mutants of $P$. gingivalis are devoid of citrullination, confirming that the citrullination of surface proteins is dependent on the activity of arginine gingipain proteases. Furthermore, recent data revealed that PPAD citrullinates peptides generated by the degradation of fibrinogen and $\alpha$-enolase by $P$. gingivalis gingipains [33]. Citrullination of fibrin and vimentin by PPAD was also described. Citrullination of bradykinin, another PPAD substrate, at the $\mathrm{C}$-terminus [31] may interfere with kinin proinflammatory activity. Finally, PPAD deiminates the C-terminal Arg in epidermal growth factor, abrogating this cytokine's biological activity [34]. PPAD is clearly expressed in vivo and is immunogenic in mice, as we were able to detect antibodies against PPAD in animals inoculated with a wild-type strain of P. gingivalis (W83) but not with the PPAD-null isogenic mutant $[35 \bullet \bullet]$.

Recent findings indicate that infection with $P$. gingivalis precedes RA and that the bacterium is a likely factor in the initiation and maintenance of the autoimmune inflammatory responses that occur in this disease [10]. In this respect, the presence of $P$. gingivalis-PAD (PPAD), an enzyme expressed by $P$. gingivalis but absent in other prokaryotes [36], may have a profound impact on the development and progression of RA via citrullination of proteins to generate neo-epitopes.

\section{Citrullination by PPAD as a Putative Mechanistic Link between PD and RA}

$P$. gingivalis is the only known microorganism to produce PAD, and in inflamed $P$. gingivalis-infested periodontitis sites, PPAD may set in motion a chain of events that breaks immunotolerance to citrullinated proteins and leads to the development of RA. In line with this hypothesis, ACPA titres in RA patients have been shown to correlate with the presence of PD [29]. Moreover, Lappin et al. recently revealed that ACPA titres are higher in patients with PD compared to healthy donors $[37 \bullet]$.

\section{Host Epitopes Modified by PPAD}

Aberrant citrullination has been observed in RA; however, it is still unclear whether citrullination in RA creates novel epitopes or uncovers cryptic ones in susceptible individuals. Among the potential autoantigens in RA that are efficiently citrullinated by human PADs are fibrinogen, enolase, vimentin, and collagen II (CII) [38, 39, 40•]. PPAD localised on the bacterial surface is perfectly positioned to citrullinate host proteins interacting with $P$. gingivalis via bacterial adhesins. In keeping with this hypothesis, $P$. gingivalis rapidly generates fibrinogen-derived peptides with carboxy-terminal citrulline residues [33]. A B cell-dominant epitope of $\alpha$ enolase termed citrullinated enolase peptide-1 (CEP-1) was also identified as an autoantigen in RA. Recent data revealed that enolase is a substrate for PPAD. Furthermore, CEP-1 shows $82 \%$ sequence similarity with $P$. gingivalis $\alpha$-enolase [41]. Therefore, CEP-1 antibodies cross-react with the equivalent epitope of $P$. gingivalis-derived enolase. An increased level of antibodies specific for CEP-1 was detected in the chamber fluid of mice infected with $P$. gingivalis [35••]. Finally, pathological changes typical of arthritis were induced in DR4-IE transgenic mice immunised with citrullinated human and $P$. gingivalis $\alpha$-enolase [40 ${ }^{\bullet}$.

\section{PPAD Autocitrullination}

P. gingivalis efficiently citrullinates its own proteins, and subcellular fractionation revealed that the majority of such proteins are associated with the periplasm, and with the outer and inner membrane fractions [33]. Although PPAD preferentially citrullinates $\mathrm{C}$-terminal arginine residues, recent data revealed a striking feature of PPAD: it undergoes autocitrullination [31, 42]. Mass spectrometry analysis revealed citrullination of 7 out of 18 arginines in the PPAD polypeptide chain, all of which were internal [32••]. Therefore, PPAD as a citrullinated bacterial protein itself constitutes a potent antigen that may break the tolerance to citrullinated host proteins. This hypothesis was recently supported by the identification of IgG specifically recognising citrullinated PPAD in sera from RA patients. A $P$. gingivalis strain that expresses catalytically inactive PPAD and is thus devoid of autocitrullination $\left(\mathrm{PPAD}^{\mathrm{C} 351 \mathrm{~A}}\right)$ did not induce an antibody response, confirming a specific immune response to citrullinated PPAD [32••]. Moreover, the significantly elevated antibody response to PPAD, but not RgpB, in sera from RA patients compared to sera from control and PD patients indicates that PPAD could be an antigen relevant to the pathogenesis of RA. The specificity of the immune response to citrullinated PPAD was confirmed by the reactivity of RA sera to multiple synthetic citrullinated peptides spanning the PPAD polypeptide chain. This study revealed the presence of antibodies to 10 of the 13

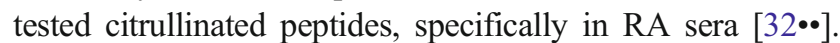
Two PPAD peptides (CPP3 and CPP8) were reactive in $40 \%$ of RA samples, indicating their possible application for screening for anti-PPAD antibodies in future epidemiological studies. Taken together, the above observations may suggest 
that $P$. gingivalis infection can uniquely prime the immune response in RA due to the appearance of autocitrullinated PPAD. The potential pathophysiologic role of autocitrullinated PPAD in RA opens up a novel area for future investigations.

\section{In Vivo Role of PPAD}

In vivo studies have revealed a relationship between joint disease and PD, as mice with pre-existing periodontitis developed more severe arthritis at a faster rate than control animals $[43,44 \cdot]$. Moreover, there is an increasing body of evidence showing that the severity of periodontitis is related to the progression of RA, and that the profile of oral microbiota in patients with new-onset RA is extremely similar to that of patients suffering from chronic RA [45, 46]. Lastly, using a collagen-induced arthritis model (CIA), it was demonstrated for the first time that infection with $P$. gingivalis not only exacerbates CIA but also appears to play a role in sensitising animals to early disease development [35••]. The arthritis that occurred in mice infected with $P$. gingivalis was characterised by significantly greater bone and cartilage destruction in the affected joints. These clinical signs of arthritis manifested significantly earlier and were accompanied by a more severe disease course than in non-infected animals. The early onset and aggravated progression of CIA occurred only in animals inoculated with viable $P$. gingivalis. Infection with $P$. intermedia, another important pathogen associated with PDs [15], had no effect on the course of CIA. Similar to infection with $P$. intermedia, inoculation with either heatkilled $P$. gingivalis or the purified cell membrane fraction from the same organism had no effect on either the rate or the severity of CIA. Taken together, these findings indicate the requirement for live $P$. gingivalis, which releases bacterial factors exerting a direct or indirect (via stimulation of host systems) effect on the host that ultimately triggers an autoimmune reaction. This hypothesis also drew on recent findings in which immunity to $P$. gingivalis, but not to $P$. intermedia (or Fusobacterium nucleatum), was shown to be significantly associated with the presence of RA-related autoantibodies in individuals at risk for the disease [47•].

Recent data revealed that chronic $P$. gingivalis oral infection prior to arthritis induction increases Th17 cell immune responses, including significant secretion of cytokines such as IL-1 $\beta$, IL-6, IL-22, tumor necrosis factor- $\alpha$, transforming growth factor- $\beta$, and IL-23. This observation suggests that chronic oral infection may influence RA development mainly through the activation of Th17-related pathways [48].

The induction of ACPA is dependent on deiminase activity; thus, PAD expressed by P. gingivalis is a potential risk factor in
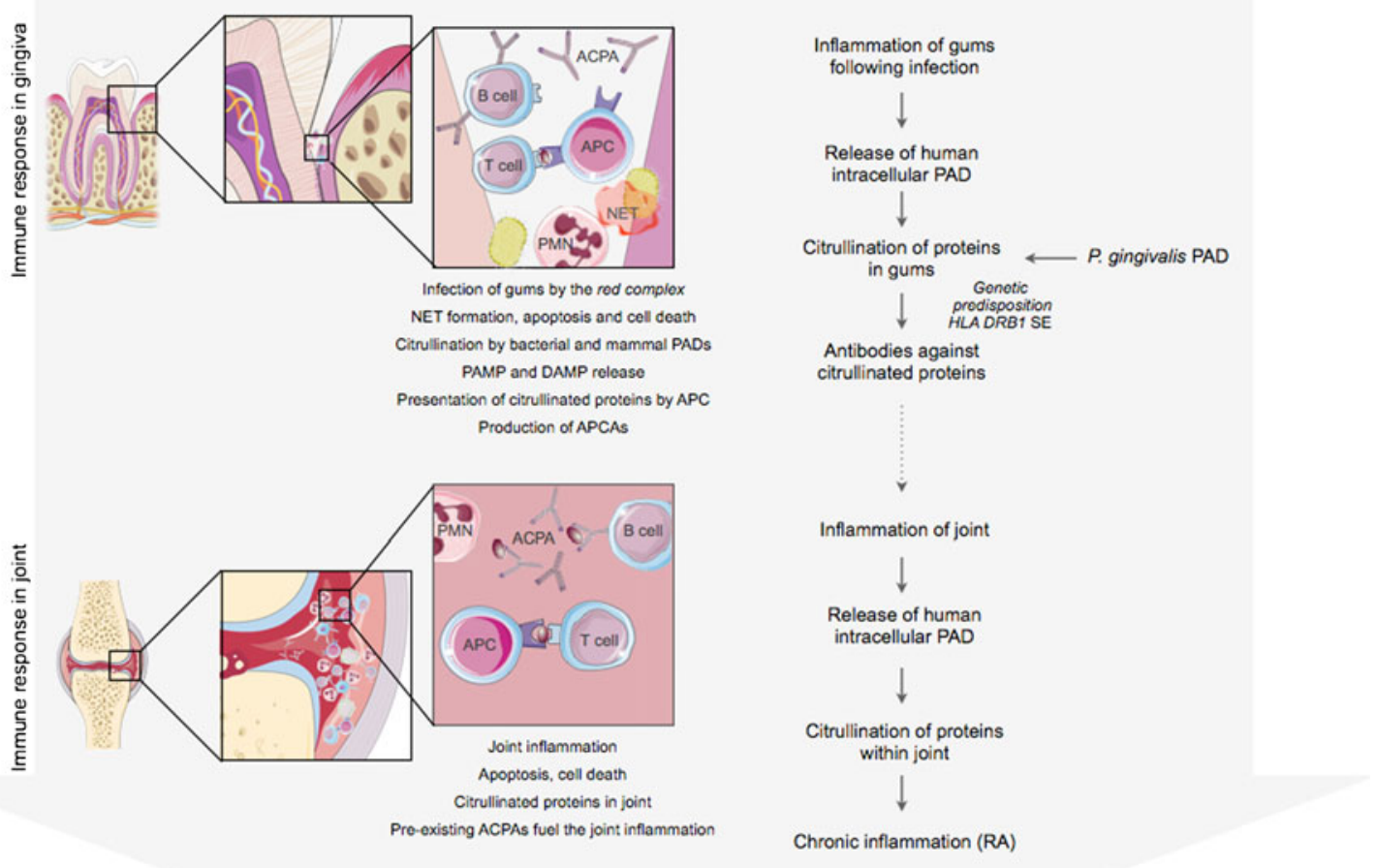

Fig. 1 A model of periodontitis-initiated pathogenesis of rheumatoid arthritis $(R A)$ initiated by PPAD/PAD-catalysed modification of proteins in the inflamed periodontal tissue and driven by autoimmunity against citrullinated epitopes joints. Figure was created using Servier Medical Art 
RA development. Recent data revealed that inoculation of animals with an isogenic $P$. gingivalis-PAD knockout strain $(\triangle \mathrm{PPAD})$ prior to immunisation with $\mathrm{CII}$ had no influence on clinical CIA development or progression [35••]. Histologically, $\triangle$ PPAD inoculation had no effect on the degree of synovitis, erosions, or neutrophil influx into the joints, as compared to CIA controls. This is in complete contrast to animals infected with the live wild-type strain (W83), suggesting that PPAD likely contributes significantly to the pathogenesis of the disease.

The enzyme by itself is immunogenic in mice, as antibodies against PPAD were detected in animals inoculated with the wild-type strain, W83 [35••]. Moreover, as mentioned before, the anti-PPAD antibody response is unique to RA patients, suggesting that PPAD may constitute a neo-antigen in RA $[32 \bullet \cdot]$. Nevertheless, it is unlikely that this response contributes to the aggravation of CIA by $P$. gingivalis because the level of anti-PPAD antibodies was very low. By contrast, the level of antibodies to citrullinated peptides $(\mathrm{aCCP})$ and to enolase-derived citrullinated epitope (CEP-1), both of which are implicated in autoimmunity in RA, strongly increased in CIA mice infected with wild-type $P$. gingivalis [35••]. Finally, demonstrating the role of autoantibody production in the pathophysiology of CIA, $P$. gingivalis infection increased the serum levels of specific IgG against CII, in contrast to either $\triangle$ PPAD or CIA control mice.

Collectively, aggravation of these RA-associated pathologies is dependent on PPAD activity, which, either directly or indirectly (via the enhancement of inflammatory reactions and the release of host PADs), leads to the generation of citrullinated neo-epitopes, thus stimulating autoantibody production. In this context, one may hypothesise that pathological citrullination of proteins in gingival tissues by PPAD, within a chronic inflammatory environment in which the immune system is stimulated by bacteria-derived danger signals such as LPS, fimbriae, and peptidoglycan, breaks immune tolerance to citrullinated proteins and peptides, priming autoimmunity in a subset of patients with RA [36] (Fig. 1).

Notably, human PADs, PPAD, and citrullinated proteins have been detected in the oral mucosa and periodontium [33]. Thus, in periodontitis, the orchestrated citrullination of host and/or bacterial proteins in vivo could trigger a loss of tolerance to structurally similar host proteins, resulting in the expression of ACPA.

\section{Conclusions}

Together, PD and RA are responsible for significant loss of function and morbidity in a large percentage of the population worldwide. Therefore, for many years, scientists have tried in vain to find a mechanistic link between both diseases in the hope of facilitating the development of a novel, effective treatment for both diseases. Based on these latest discoveries, we believe that PPAD could be a target for successful therapy or prevention of these crippling diseases.

Acknowledgments This work was supported by grants from the EC (FP7-HEALTH-2010-261460 "Gums \& Joints", FP7-HEALTH-F32012-306029 "TRIGGER”, and FP7-PEOPLE-2011-ITN-290246 "RAPID"), the National Institutes of Health/NIDCR, USA (grant DE 09761), National Science Center, Poland (UMO-2011/01/B/NZ6/00268, 2012/04/A/NZ1/00051 to Jan Potempa and UMO-2011/03/B/NZ6/00053 to Joanna Koziel), the Foundation for Polish Science (TEAM project DPS/424-329/10), and Polish Ministry of Science and Higher Education (137/7.PR-EU/2011/2). The Faculty of Biochemistry, Biophysics and Biotechnology of the Jagiellonian University is a beneficiary of structural funds from the European Union (POIG.02.01.00-12-064/08).

\section{Compliance with Ethics Guidelines}

Conflict of Interest Joanna Koziel, Piotr Mydel, and Jan Potempa declare that they have no conflict of interest.

Human and Animal Rights and Informed Consent This article does not contain any studies with human or animal subjects performed by any of the authors.

Open Access This article is distributed under the terms of the Creative Commons Attribution License which permits any use, distribution, and reproduction in any medium, provided the original author(s) and the source are credited.

\section{References}

Papers of particular interest, published recently, have been highlighted as:

- Of importance

.. Of major importance

1. Firatli E, Kantarci A, Cebeci I, Tanyeri H, Sonmez G, Carin M, et al. Association between HLA antigens and early onset periodontitis. J Clin Periodontol. 1996;23:563-6.

2. Katz J, Goultschin J, Benoliel R, Brautbar C. Human leukocyte antigen (HLA) DR4. Positive association with rapidly progressing periodontitis. J Periodontol. 1987;58:607-10.

3. Marotte H, Farge P, Gaudin P, Alexandre C, Mougin B, Miossec P. The association between periodontal disease and joint destruction in rheumatoid arthritis extends the link between the HLA-DR shared epitope and severity of bone destruction. Ann Rheum Dis. 2006;65:905-9.

4. Bonfil JJ, Dillier FL, Mercier P, Reviron D, Foti B, Sambuc R, et al. A "case control" study on the role of HLA DR4 in severe periodontitis and rapidly progressive periodontitis. Identification of types and subtypes using molecular biology (PCR.SSO). J Clin Periodontol. 1999;26:77-84.

5. de Pablo P, Dietrich T, McAlindon TE. Association of periodontal disease and tooth loss with rheumatoid arthritis in the US population. J Rheumatol. 2008;35:70-6.

6. Berthelot JM, Le Goff B. Rheumatoid arthritis and periodontal disease. Joint Bone Spine. 2010;77:537-41.

7. Demmer RT, Molitor JA, Jacobs Jr DR, Michalowicz BS. Periodontal disease, tooth loss and incident rheumatoid arthritis: results from the First National Health and Nutrition Examination 
Survey and its epidemiological follow-up study. J Clin Periodontol. 2011;38:998-1006.

8. Detert J, Pischon N, Burmester GR, Buttgereit F. The association between rheumatoid arthritis and periodontal disease. Arthritis Res Ther. 2010;12:218.

9. Rutger Persson G. Rheumatoid arthritis and periodontitis - inflammatory and infectious connections. Review of the literature. J Oral Microbiol. 2012;4:11829-11845

10. Lundberg K, Wegner N, Yucel-Lindberg T, Venables PJ. Periodontitis in RA-the citrullinated enolase connection. Nat Rev Rheumatol. 2010;6:727-30.

11. Demmer RT, Papapanou PN. Epidemiologic patterns of chronic and aggressive periodontitis. Periodontology. 2010;2000(53):28-44.

12. Kebschull M, Demmer RT, Papapanou PN. "Gum bug, leave my heart alone!"-epidemiologic and mechanistic evidence linking periodontal infections and atherosclerosis. J Dent Res. 2010;89:879-902.

13. de Pablo P, Chapple IL, Buckley CD, Dietrich T. Periodontitis in systemic rheumatic diseases. Nat Rev Rheumatol. 2009;5:218-24.

14. Farquharson D, Butcher JP, Culshaw S. Periodontitis, Porphyromonas, and the pathogenesis of rheumatoid arthritis. Mucosal Immunol. 2012;5:112-20.

15. Socransky SS, Haffajee AD, Cugini MA, Smith C, Kent Jr RL. Microbial complexes in subgingival plaque. J Clin Periodontol. 1998;25:134-44.

16. Hajishengallis G, Liang S, Payne MA, Hashim A, Jotwani R, Eskan MA, et al. Low-abundance biofilm species orchestrates inflammatory periodontal disease through the commensal microbiota and complement. Cell Host Microbe. 2011;10:497-506.

17. Guo Y, Nguyen KA, Potempa J. Dichotomy of gingipains action as virulence factors: from cleaving substrates with the precision of a surgeon's knife to a meat chopper-like brutal degradation of proteins. Periodontology. 2010;2000(54):15-44.

18. Krauss JL, Potempa J, Lambris JD, Hajishengallis G. Complementary Tolls in the periodontium: how periodontal bacteria modify complement and Toll-like receptor responses to prevail in the host. Periodontology. 2010;2000(52):141-62.

19. Potempa M, Potempa J. Protease-dependent mechanisms of complement evasion by bacterial pathogens. Biol Chem. 2012;393:873-88.

20. Ford PJ, Gamonal J, Seymour GJ. Immunological differences and similarities between chronic periodontitis and aggressive periodontitis. Periodontology. 2010;2000(53):111-23.

21. Liu YC, Lerner UH, Teng YT. Cytokine responses against periodontal infection: protective and destructive roles. Periodontology. 2010;2000(52):163-206.

22. Klareskog L, Catrina AI, Paget S. Rheumatoid arthritis. Lancet. 2009;373:659-72.

23. Klareskog L, Ronnelid J, Lundberg K, Padyukov L, Alfredsson L. Immunity to citrullinated proteins in rheumatoid arthritis. Annu Rev Immunol. 2008;26:651-75.

24. Wegner N, Lundberg K, Kinloch A, Fisher B, Malmstrom V, Feldmann M, et al. Autoimmunity to specific citrullinated proteins gives the first clues to the etiology of rheumatoid arthritis. Immunol Rev. 2010;233:34-54.

25. Gyorgy B, Toth E, Tarcsa E, Falus A, Buzas EI. Citrullination: a posttranslational modification in health and disease. Int $\mathrm{J}$ Biochem Cell Biol. 2006;38:1662-77.

26. Wang Y, Li M, Stadler S, Correll S, Li P, Wang D, et al. Histone hypercitrullination mediates chromatin decondensation and neutrophil extracellular trap formation. J Cell Biol. 2009;184: 205-13.

27. van Gaalen F, Ioan-Facsinay A, Huizinga TW, Toes RE. The devil in the details: the emerging role of anticitrulline autoimmunity in rheumatoid arthritis. J Immunol. 2005; 175:5575-80.

28. Hendler A, Mulli TK, Hughes FJ, Perrett D, Bombardieri M, HouriHaddad Y, et al. Involvement of autoimmunity in the pathogenesis of aggressive periodontitis. J Dent Res. 2010;89:1389-94.
29. Dissick A, Redman RS, Jones M, Rangan BV, Reimold A, Griffiths GR, et al. Association of periodontitis with rheumatoid arthritis: a pilot study. J Periodontol. 2010;81:223-30.

30. Vossenaar ER, Zendman AJ, van Venrooij WJ, Pruijn GJ. PAD, a growing family of citrullinating enzymes: genes, features and involvement in disease. Bioessays. 2003;25:1106-18.

31. McGraw WT, Potempa J, Farley D, Travis J. Purification, characterization, and sequence analysis of a potential virulence factor from Porphyromonas gingivalis, peptidylarginine deiminase. Infect Immun. 1999;67:3248-56.

32.• Quirke AM, Lugli EB, Wegner N, Hamilton BC, Charles P, Chowdhury M, et al. Heightened immune response to autocitrullinated Porphyromonas gingivalis peptidylarginine deiminase: a potential mechanism for breaching immunologic tolerance in rheumatoid arthritis. Ann Rheum Dis. 2013;73:263-9. Established specific immune response to PPAD what might break tolerance in $R A$.

33. Wegner N, Wait R, Sroka A, Eick S, Nguyen KA, Lundberg K, et al. Peptidylarginine deiminase from Porphyromonas gingivalis citrullinates human fibrinogen and alpha-enolase: implications for autoimmunity in rheumatoid arthritis. Arthritis Rheum. 2010;62: 2662-72.

34. Pyrc K, Milewska A, Kantyka T, Sroka A, Maresz K, Koziel J, et al. Inactivation of epidermal growth factor by Porphyromonas gingivalis as a potential mechanism for periodontal tissue damage. Infect Immun. 2012;81:55-64.

35.• Maresz KJ, Hellvard A, Sroka A, Adamowicz K, Bielecka E, Koziel J, et al. Porphyromonas gingivalis facilitates the development and progression of destructive arthritis through its unique bacterial peptidylarginine deiminase (PAD). PLoS Pathog. 2013;9:e1003627. The first paper indicating in vivo the role of $P$. gingivalis PPAD in RA developing and progression.

36. Mangat $\mathrm{P}$, Wegner N, Venables PJ, Potempa J. Bacterial and human peptidylarginine deiminases: targets for inhibiting the autoimmune response in rheumatoid arthritis? Arthritis Res Ther. 2010;12:209.

37. Lappin DF, Apatzidou D, Quirke AM, Oliver-Bell J, Butcher JP, Kinane DF, et al. Influence of periodontal disease, Porphyromonas gingivalis and cigarette smoking on systemic anti-citrullinated peptide antibody titres. J Clin Periodontol. 2013;40:907-15. The paper documents increase of ACPA in PD patients in comparison to healthy donors.

38. Gilliam BE, Reed MR, Chauhan AK, Dehlendorf AB, Moore TL. Evidence of fibrinogen as a target of citrullination in IgM rheumatoid factor-positive polyarticular juvenile idiopathic arthritis. Pediatr Rheumatol Online J. 2011;9:8.

39. Harre U, Georgess D, Bang H, Bozec A, Axmann R, Ossipova E, et al. Induction of osteoclastogenesis and bone loss by human autoantibodies against citrullinated vimentin. J Clin Invest. 2012;122:1791-802.

40. Kinloch AJ, Alzabin S, Brintnell W, Wilson E, Barra L, Wegner N, et al. Immunization with Porphyromonas gingivalis enolase induces autoimmunity to mammalian alpha-enolase and arthritis in DR4-IEtransgenic mice. Arthritis Rheum. 2011;63:3818-23. Presents the first animal model that links an immune response to $P$ gingivalis enolase to an important subset of $R A$, defined by antibodies to citrullinated $\alpha$-enolase in the context of DR4.

41. Lundberg K, Kinloch A, Fisher BA, Wegner N, Wait R, Charles P, et al. Antibodies to citrullinated alpha-enolase peptide 1 are specific for rheumatoid arthritis and cross-react with bacterial enolase. Arthritis Rheum. 2008;58:3009-19.

42. Rodriguez SB, Stitt BL, Ash DE. Expression of peptidylarginine deiminase from Porphyromonas gingivalis in Escherichia coli: enzyme purification and characterization. Arch Biochem Biophys. 2009;488:14-22.

43. Bartold PM, Marino V, Cantley M, Haynes DR. Effect of Porphyromonas gingivalis-induced inflammation on the development of rheumatoid arthritis. J Clin Periodontol. 2010;37:405-11. 
44. Cantley MD, Haynes DR, Marino V, Bartold PM. Pre-existing periodontitis exacerbates experimental arthritis in a mouse model. J Clin Periodontol. 2011;38:532-41. An in vivo study indicating that pre-existing $P$. gingivalis induced periodontitis exacerbated experimental arthritis in a mouse model.

45. Scher JU, Ubeda C, Equinda M, Khanin R, Buischi Y, Viale A, et al. Periodontal disease and the oral microbiota in new-onset rheumatoid arthritis. Arthritis Rheum. 2012;64:3083-94.

46. Smit MD, Westra J, Vissink A, Doornbos-van der Meer B, Brouwer E, van Winkelhoff AJ. Periodontitis in established rheumatoid arthritis patients: a cross-sectional clinical, microbiological and serological study. Arthritis Res Ther. 2012;14:R222.
47. Mikuls TR, Thiele GM, Deane KD, Payne JB, O'Dell JR, Yu F, et al. Porphyromonas gingivalis and disease-related autoantibodies in individuals at increased risk of rheumatoid arthritis. Arthritis Rheum. 2012;64:3522-30. Immunity to $P$ gingivalis, but not other periodontopathogens is significantly associated with the presence of $R A$-related autoantibodies in individuals at risk of $R A$, which may suggest that infection with $P$ gingivalis plays a central role in the early loss of tolerance to self-antigens that occur in the pathogenesis of RA.

48. Marchesan JT, Gerow EA, Schaff R, Taut AD, Shin SY, Sugai J, et al. Porphyromonas gingivalis oral infection exacerbates the development and severity of collagen-induced arthritis. Arthritis Res Ther. 2013;15:R186 